\title{
ESTUDIO EMPÍRICO SOBRE EL ESTADO Y TENDENCIAS DE LA RESPONSABILIDAD SOCIAL CORPORATIVA EN ECUADOR MEDIANTE EL ANÁLSIS DE CASO: DEL CANTÓN DE LOJA
}

\author{
Mónica-Patricia Costa-Ruiz ${ }^{1}$ \\ Verónica-Alexandra Armijos-Buitrón ${ }^{2}$ \\ Jhoana-Elizabeth Paladines-Benítez ${ }^{3}$ \\ Valentín-Alejandro Martínez-Fernández ${ }^{4}$ \\ Universidad Técnica Particular de Loja - Ecuador
}

\begin{abstract}
${ }^{1}$ Magister Gestión Empresarial por la Universidad Técnica Particular de Loja (UTPL) Ecuador. Especialista en Economía Popular y Solidaria por la Universidad Nacional de Loja y HEGOA Ecuador-España, Economista por la Universidad Técnica Particular de Loja. Coordinadora de la maestría de Gestión de la Responsabilidad Social Corporativa de la UTPL. Docente titular de la UTPL. Forma parte del grupo de investigación Innovación y Nueva Empresa. e-mail: mpcosta@utpl.edu.ec
\end{abstract}

${ }^{2}$ Magister en Dirección y Administración de Empresas por el Instituto de Estudios Bursátiles IEB de Madrid España y Master en Gestión de la Formación Planificación, Desarrollo y Evaluación de la Formación de Formadores por la Universidad de Sevilla - España. Ingeniero Comercial por la Escuela Politécnica del Ejército -ESPE- Ecuador. Doctoranda en Administración de Empresas por la Universidad Nacional de Rosario Argentina. Docente Principal de la Universidad Técnica Particular de Loja, miembro del Equipo de Calidad de la Titulación de Administración de Empresas de la UTPL. Forma parte del grupo de investigación Innovación y Nueva Empresa. e-mail: vaarmijos@utpl.edu.ec

${ }^{3}$ Magister en Educación a Distancia de la UTPL- Loja, Ecuador. Doctoranda en Administración de Empresas de la Universidad Nacional de Rosario- Rosario- Argentina, Licenciada en Ciencias de la Educación, mención Inglés de la Universidad Técnica Particular de Loja (UTPL)-Ecuador, Ingeniera en Administración de Empresas de la UTPL- Ecuador, Experta en nuevas tecnologías de Aprendizaje a través de Internet de la Universidad Politécnica de Furtwangen, Alemania. Diploma en Fundamentos en Educación a Distancia- UTPL- Loja, Ecuador, Especialista en Planificación Curricular y Organización de Sistemas de Educación a Distancia-.UTPLEcuador, Docente- Investigadora, UTPL. Miembro del Consejo Directivo de la Red Latinoamericana Educal. Forma parte del grupo de investigación Innovación y Nueva Empresa. e-mail: jepaladines@utpl.edu.ec

${ }^{4}$ Es profesor Titular de Comercialización en Investigación de Mercados de la Universidad de A Coruña. Doctor en Ciencias de la Información y Licenciado en Ciencias de la Información por la Universidad Complutense de Madrid. Máster en Dirección y Administración de Empresas (MBA) por la Universidad de la Coruña. Es coordinador e investigador principal del Grupo de Investigación en Marketing Aplicado de la Universidad de la Coruña.e-mail: valejand@udc.es

5 Este trabajo forma parte de la contribución al proyecto Prometeo-Senescyt (con vinculación No. PROMETEO-CEB-008-2015)

REDMARKA UIMA-Universidad de A Coruña - CIECID

Año VIII, Número 15, (2015), v I pp. 43-67

http://www.redmarka.net/ ISSN 1852-2300 
Material original autorizado para su primera publicación en la revista académica REDMARKA. Revista Digital de Marketing Aplicado.

https://doi.org/10.17979/redma.2015.01.015.4878

Recibido: 4 Mayo 2015

Aceptado 17 Septiembre 2015

\section{RESUMEN}

La Responsabilidad Social Corporativa (RSC) en Ecuador es un concepto de reciente aplicación,sin embargo, es un tema que en los últimos años ha ganado importancia y consolidado como una tendencia de gestión empresarial.

Ante esta realidad, se efectuó un estudio de caso en el cantón Loja, de carácter aproximativo y experimental, a fin de analizar la situación actual respecto a la aplicación de las prácticas de RSC en los tres sectores económicos. Se aplicó una encuesta a 222 empresas, aplicando el método de muestreo aleatorio simple. Se utilizó un cuestionario diseñado por el instituto ETHOS de Brasil, y aplicado por la Asociación de Empresas Cristianas (ADEC) de Paraguay, éste fue ajustado a las características del lugar de investigación.

El análisis efectuado con los últimos datos consolidados por el Instituto Nacional de Estadísticas y Censos de Ecuador (INEC), con información secundaria y al 2013, refleja que en Loja existían 18.025 empresas activas. El sector con mayor representatividad es el terciario, con 15.604 empresas; el sector secundario con 1.500; y, el sector primario con 921. Los tres sectores alcanzaron un nivel aproximado de ventas de 1.034 (millones de dólares), contribuyendo el sector terciario con el $92 \%$ del total, el secundario con el $6 \%$ y el primario con el $2 \%$; de igual forma emplearon a 48.061 personas, el $54 \%$ fueron hombres y el $46 \%$ mujeres.

Los objetivos de esta investigación se alcanzaron analizando indicadores por cada grupo de interés y respectivamente fueron evaluados de acuerdo al cumplimiento de las diferentes prácticas integradas en aquellos. Los resultados reflejan que, en relación al 
compromiso ético, los tres sectores tienen una aplicación del $70 \%$ de las prácticas relacionadas con este indicador; el sector primario muestra un mayor diálogo e implicación con los grupos de interés, con un cumplimiento del $90 \%$; y, con carácter general, en los veintitrés indicadores estudiados se observó que el sector primario es el que aplica en mayor medida las diferentes prácticas de RSC, y el sector terciario el de menor cumplimiento. El análisis estadístico determinó que el tamaño, edad y sector no son factores determinantes en la aplicación de la RSC.

Palabras clave: Responsabilidad Social Corporativa, sectores económicos, indicadores.

\section{ABSTRACT}

Corporate Social Responsibility (CSR) in Ecuador is a new concept applied in national companies; however it is an issue that in recent years has gained prominence and established itself as a trend of business management.

That is why; a case study was conducted in Loja city, this study was rough and experimental; the aim of it was to analyze the current situation regarding the implementation of CSR practices in the three sectors of the economy. For carrying out the study a survey was applied to a sample of 222 companies, the sample was calculated by the method of simple random sampling. The used instrument was a questionnaire designed by ETHOS Institute of Brazil; it was implemented and validated by the Association of Christian Businesses (ADEC) of Paraguay, for this study it was adjusted to the characteristics of the research.

Also for the research secondary information was used, it was taken of Instituto Nacional de Estadisticas y Censos de Ecuador (INEC) (2013), and it shows that in Loja were 18.025 active companies. The largest sector is the tertiary, with 15.604 companies; the secondary sector with 1.500; and the primary sector with 921. Together the three sectors reached a sales level of about 1,034 million; the tertiary sector contributed $92 \%$ of total sales, with $6 \%$ secondary and primary with $2 \%$. Similarly, the three sectors employed 48.061 workers of which $54 \%$ were men and $46 \%$ women.

To achieve the objectives of this research, indicators were analyzed for each stakeholder and they were evaluated according to the fulfillment of the CSR practices. The results 
show that, in relation to ethical commitment indicator, the three sectors have an application of the $70 \%$ of practices; related to dialogue and engagement with stakeholders indicator the primary sector shows a $90 \%$ of application and, in general, in the twenty three indicators studied it was observed that the primary sector is the one that applies in a greatest way the different CSR practices, and the tertiary sector the lowest one. From the regression analysis it was found that the variables size, age and industry are not determining factors in implementing CSR practices.

Keywords: Corporate Social Responsibility, economic sectors, indicators.

\section{INTRODUCCIÓN}

Cuando nace el concepto de Responsabilidad Social Corporativa (RSC) también las empresas se cuestionan si ellas tienen o no responsabilidades con la sociedad (clientes, proveedores, empleados, gobierno, medio ambiente y comunidad). Para algunos autores la respuesta es afirmativa, aunque también existe una corriente en la cual se considera que el único papel que desempeña la empresa en la sociedad consiste en la generación de utilidades para los accionistas. Sin embargo, esta obligación de actuar responsablemente fue reconocida por Bowen en 1953, quien propone las primeras concepciones de la RSC.

En la actualidad se puede decir que este tema ha cobrado mayor importancia, pues la empresa se desarrolla en un entorno en el que se genera conciencia por el medio ambiente y la sociedad, de modo que aquellas deben de responder a esas inquietudes a través de la puesta en marcha de prácticas de RSC.

La RSC puede ser asumida por las empresas como un modelo de gestión empresarial, que contribuya al desarrollo económico sustentable a través de la incorporación del dialogo con los grupos de interés. La importancia que se otorga a dichos grupos ha de reflejarse en la misión de la empresa para generar una cultura sólida. 
En este sentido, surge el estudio reflejado en este artículo con la finalidad de exponer la situación actual de la RSC en el cantón Loja; para lo cual se desarrolló un trabajo de investigación como parte de un proyecto financiado por la Universidad Técnica Particular de Loja, y cuya finalidad es probar la metodología planteada para replicar en futuras investigaciones a nivel nacional.

La investigación se fundamenta en el desarrollo del estado del arte de la RSC, mostrando los aportes teóricos de varios autores, así como la evolución que el concepto de RSC ha tenido a lo largo de los últimos años. Es importante mencionar que durante la etapa de la revisión bibliográfica se evidenció que existe escasa información sobre la aplicación de prácticas de RSC en el Ecuador.

Se incluye la caracterización económica del cantón Loja, y en base a la cual se desarrolló el estudio de acuerdo a los objetivos planteados, finalmente se muestra los resultados obtenidos a través del contraste de las hipótesis propuestas.

\section{ESTADO DEL ARTE}

\subsection{Responsabilidad Social Corporativa}

\subsubsection{Evolución del concepto de Responsabilidad Social Corporativa (RSC) ${ }^{6}$}

El concepto de Responsabilidad Social Corporativa, como consecuencia del entorno dinámico en el cual se desarrolla, ha experimentado significativos cambios de enfoque en las dos últimas décadas y cuyo punto de partida puede situarse en las aproximaciones realizadas por Bowen, Davis y Bloomstrom, en 1953, hasta las delimitaciones efectuadas por Carrol, en 1979, decurso en el cual se presentan los principales aportes teóricos que, a su vez, sirven de base para el análisis histórico-conceptual que se expone a continuación, a modo de marco de referencia, para comprender las actuales claves explicativas de esta forma de entender las relaciones entre la empresa y la sociedad en la cual se encuentra incursa su actividad.

\footnotetext{
${ }^{6}$ Información tomada de García González (2012)
} 
La Responsabilidad social corporativa tiene sus inicios en 1953 con la definición propuesta por Howard Bowen, al considerar que la misma "se refiere a las obligaciones de los hombres de negocios, respecto a seguir políticas, tomar decisiones, o seguir líneas de actuación que son deseables en términos de los objetivos y valores de nuestra sociedad".

Kate Davis, en 1960, cuestionaba abiertamente si las empresas podían permitirse ignorar sus responsabilidades sociales, y así junto a Robert Bloomstrom presentan una aproximación al concepto de responsabilidad social, conforme al cual ésta consiste esencialmente en "la obligación de una persona en cuanto a considerar los efectos de sus decisiones y de sus acciones sobre el conjunto del sistema social"; Bloomstrom completa la definición, al añadir que "la sustancia de la responsabilidad social surge de la preocupación por las consecuencias éticas de los propios actos, dado que éstos pueden afectar a los intereses de otros [...]"

Sería Walton quien, en 1967, matizara que el concepto de responsabilidad social reconoce el carácter íntimo de las relaciones entre la corporación y la sociedad; al tiempo de subrayar que tales relaciones deben de ser tomadas en cuenta por los altos directivos, en tanto la corporación y los grupos con ella relacionados alcancen sus respectivos objetivos. El mismo autor junto con Eells, en 1974, establece que la Responsabilidad Social Corporativa supone una preocupación por las necesidades y objetivos de la sociedad que va más allá de lo meramente económico ... por el papel de las empresas de cara a apoyar y mejorar el orden social.

Johnson, en 1971, presenta cuatro enfoques de Responsabilidad Social Corporativa: a) convencional, b) de maximización de la utilidad, c) maximización de beneficios a largo plazo; y, d) el enfoque lexicográfico. Será el enfoque convencional el de mayor aporte, al precisar que una empresa socialmente responsable es aquella cuyo personal directivo equilibra una multiplicidad de intereses. En vez de luchar sólo por lograr mayores beneficios para sus accionistas, una empresa responsable también ha de tener en cuenta a los empleados, a los proveedores, a los distribuidores, a las comunidades locales y, a la nación; con lo cual dio como resultado la aparición de la teoría de los grupos de interés (stakeholders). 
Por su parte, Carroll define ya, en 1979, la Responsabilidad Social corporativa como la actividad empresarial que armoniza las expectativas económicas, legales, éticas y discrecionales que la sociedad tiene sobre las organizaciones en un momento de tiempo dado. A partir de esta nueva visión, se consolida la idea de que la Responsabilidad Social Corporativa es de carácter voluntario, por ende proactiva, y va más allá de la ley y la incorporación de valores y expectativas sociales en la gestión empresarial.

Por otro lado, Martínez y Rodríguez (2013) explican que el modelo de Carroll se sostiene en cuatro dimensiones que, a su vez, dependen sucesivamente unas de otras. En primer lugar, la dimensión económica se refiere a las expectativas de la sociedad en cuanto a que las empresas sean rentables y obtengan utilidades (como incentivo y recompensa a su eficiencia y eficacia) mediante la producción y venta de bienes y servicios. La dimensión legal, entendida como las expectativas de la sociedad de que las empresas alcancen sus objetivos económicos circunscribiéndose al marco jurídico establecido. En tercer lugar, la dimensión ética se refiere a las expectativas de la sociedad de que el comportamiento y las prácticas empresariales satisfagan ciertas normas éticas. Finalmente, la dimensión discrecional o filantrópica, relativa a las expectativas de la sociedad de que las empresas se impliquen voluntariamente en actividades que satisfagan las normas sociales.

De acuerdo a la revisión conceptual efectuada, puede colegirse que la definición de la Responsabilidad Social Corporativa ha experimentado sustanciales cambios, en un proceso de orden evolutivo, de modo que en cada uno de los nuevos aportes formulados por los autores citados se han señalado características relevantes a este concepto y, de acuerdo a una visión analítica de las mismas, se puede concluir sintéticamente que la RSC es:

$\checkmark$ Una obligación de los hombres de negocios de adoptar decisiones encaminadas a cumplir con los valores de una sociedad.

$\checkmark$ Un reconocimiento del vínculo entre la empresa y la sociedad. 
$\checkmark$ Constituye una preocupación por las necesidades y objetivos de la sociedad que va más allá de lo meramente económico.

$\checkmark$ Analiza la importancia de los empleados, los proveedores, los distribuidores, las comunidades locales y, la nación.

$\checkmark$ Una actividad empresarial que armoniza las expectativas económicas, legales, éticas y discrecionales de la organización con la sociedad.

La RSC es en sí misma una estrategia de gestión empresarial que permite un relacionamiento exitoso de la empresa con la sociedad y el medioambiente, al tiempo de generar la consolidación de la misma en el mercado; y, por consiguiente, facilita mejorar el modelo productivo al incorporar criterios de ética, eficiencia y sostenibilidad.

\subsubsection{La RSC en la actualidad.}

De acuerdo a la Comisión de las Comunidades Europeas (2001), actualmente la responsabilidad social se entiende como la integración voluntaria, por parte de las empresas, de las preocupaciones sociales, y medioambientales en sus operaciones comerciales y sus relaciones con sus interlocutores.

En este sentido, Diez de Castro et al (2001) consideran que la responsabilidad social es una postura proactiva, o una "obligación voluntaria" que adoptan las empresas, con respecto a los grupos o agentes sociales con los que interactúa, de manera que sus comportamientos estén orientados por criterios éticos. La Responsabilidad Social implica el compromiso de no provocar daños con sus acciones, así como colaborar con el desarrollo de la sociedad dentro de un marco ético.

Por su parte, Bateman y Snell (2004) definen a la Responsabilidad Social Corporativa como la obligación hacia la sociedad que asumen las empresas. La compañía socialmente responsable maximiza sus efectos positivos sobre la sociedad y minimiza sus efectos negativos. 
Steurer (2005), citado en Orquera (2013), estima que la Responsabilidad Social Corporativa busca integrar las preocupaciones sociales y del medio ambiente en las operaciones comerciales de las empresas, así como en las relaciones de las organizaciones con los stakeholders de manera voluntaria.

Dávila y Gómez (2008), citado por Tejedor (2015), señalan que a fin garantizar de las empresas la asunción de una verdadera RSC, es necesario diferenciar las dimensiones internas y externas que componen a toda organización. Desde el punto de vista interno, se encuentran los siguientes aspectos: a) gestión de recursos humanos, b) salud y seguridad en el lugar de trabajo, c) adaptación al cambio y d) gestión del impacto ambiental y de los recursos naturales.

Raufflet et al (2012) concluyen que el periodo comprendido entre el 2000 y 2010, es cuando la Responsabilidad Social Corporativa incorpora como uno de sus objetivos esenciales la búsqueda de la sostenibilidad, y se da una convergencia entre estos dos conceptos. Por un lado, el desarrollo sostenible vinculado con actores de índole público o social; especialmente con grupos preocupados por el medio ambiente; y, por otro, la RSC en el sector privado, que busca mejorar las relaciones con sus grupos de interés y reducir su impacto ambiental.

A raíz de esta convergencia, surgen iniciativas de diferentes organismos internacionales interesados en la promoción del desarrollo, a través del reconocimiento de la RSC como un elemento clave para la consecución de este objetivo.

Por su parte, Argandoña (2012) realiza una reflexión sobre lo que es y no es la responsabilidad social, argumentando principalmente que la RS no es una técnica de gestión o un conjunto de prácticas o de herramientas, si no que constituye la razón de ser de la empresa y a su vez ésta necesita de instrumentos de ejecución para que la RS no quede en buenas intenciones. Cuestiona también que la RS no debe partir de un modelo abstracto propuesto por los expertos, si no del contexto en el que se encuentra cada empresa en cada momento, estableciendo un dialogo permanente entre propietarios, directivos, empleados y otros stakeholders. 
Como conclusión, Martínez y Rodríguez (2013) sostienen que, desde un punto de vista empresarial y directivo, ha de remarcarse que la RSC no se trata de programas de filantropía y donación, pues se trata de una serie de estrategias y políticas que no son fáciles de gestionar y controlar, construyéndose en torno a un contexto y a unas circunstancias cambiantes con gran influencia en la trayectoria presente y futura de la organización, ya que han de tener en cuenta las expectativas de diversos grupos de interés.

\subsubsection{Diferencias entre Comportamiento Ético en las empresas y Responsabilidad Social Corporativa.}

Los diferentes agentes que intervienen en el mercado exigen también de las empresas un comportamiento que trascienda el ámbito meramente legal y también el propio de la Responsabilidad Social Corporativa. Una forma de hacer, de actuar que responda a una actitud y se sitúe en el plano de la moral e integrada en la concepción de la Ética. Por ello, las empresas para poder mantener una posición socialmente aceptable han de ser reconocidas por sus púbicos como organizaciones éticas y consecuentemente mostrar una gestión transparente; esto logrará un acomodo adecuado de las actividades empresariales a la moral social y generar una percepción de lo bueno que incida en la construcción de una reputación positiva para lograr una importante ventaja competitiva en un entorno que se encuentra dinamizado por la actividad corporativa en constante desarrollo.

Las conductas directivas cuando no se encuadran en un comportamiento ético, generan conflictos dentro de la organización, lo que repercute en el desenvolvimiento de las actividades de cada miembro de la misma, pues se ve afectada la armonía en el trabajo y genera así mismo desconfianza y falta de compromiso con los objetivos a corto, mediano y largo plazo que la empresa busca cumplir.

Al estar íntimamente ligada la ética y la moral, al ser concebidas como universales, no es posible diferenciar situaciones éticas en la empresa que se deban de distinguir entre otros 
escenarios; por el contrario, la ética empresarial busca dar las pautas generales sobre las cuales las empresas han de encaminar sus acciones para el cumplimiento de sus estrategias planificadas.

Camejo (2010) afirma que las empresas modernas en un mundo globalizado, como el de hoy, han de reconocer que tienen responsabilidades y las mismas van más allá del simple cumplir con la ley, maximizar el retorno hacia los accionistas, o hacer filantropía.

De este modo, las empresas que cumplen con las prácticas de Responsabilidad Social Corporativa están enmarcadas dentro de sus obligaciones éticas. Pero, ser moralmente responsable es ir más allá, lograr una armonía sistémica entre las acciones de todos los involucrados en la actividad empresarial, conjugar las responsabilidades del conjunto de los grupos de interés para lograr una convivencia armónica dirigida a posibilitar el desarrollo del ser humano que coexiste dentro de la sociedad.

La moralidad de la sociedad vincula la actividad de la empresa como medio para mejorar las condiciones de vida en comunidad. La empresa, al ser el resultado de una combinación de factores sociales, muestra cierta propensión a enfrentar conflictos de diferente orden, tanto sociales, como culturales, todos ellos relacionados con su gestión de productividad; por ello, la ética se concatena en todas las áreas con el fin de buscar, desde las diferentes directrices, cumplir con su rol de organización con sensibilidad social para poder atender los diferentes enfoques de la Responsabilidad Social Corporativa, como medio de vincular a los grupos de interés hacia una verdadera transformación social que procure la modulación adecuada entre lo social, lo económico y lo ambiental.

Las estrategias empresariales deberán estar alineadas bajo un contexto de moralidad y ética, de esta manera los resultados obtenidos se conjugarán de forma adecuada con las buenas prácticas de Responsabilidad Social Corporativa, política de desarrollo económico y social bajo el cual la administración moderna busca plasmar su visión global de sustentabilidad y trabajo.

\subsubsection{Responsabilidad Social corporativa en Ecuador}


La Responsabilidad Social Corporativa en Ecuador es un concepto emergente en las empresas nacionales. No obstante, es un enfoque de gestión que gana importancia y se abre camino como una nueva tendencia de la relación de la empresa con su entorno dentro del territorio ecuatoriano y que, en cierto modo, provoca un cambio significativo en la percepción de los públicos de la acción empresarial al entenderla como algo que contribuye de manera positiva no sólo al desarrollo económico, sino también al social y cultural del conjunto de la ciudadanía.

Consecuentemente con la importancia y proyección de la Responsabilidad Social en Ecuador, son ya dos las organizaciones que, a nivel nacional, se centran en analizar e investigar todo aquello relacionado, directa o indirectamente, con la RSC: el Consorcio Ecuatoriano para la Responsabilidad Social (CERES) y el Instituto de Responsabilidad Social Empresarial (IRSE).

Cabe precisar que CERES es una red compuesta por empresas públicas, privadas, fundaciones empresariales y organizaciones de la sociedad civil, comprometidas con la sostenibilidad social, ambiental y económica.

Por su parte, el IRSE es una organización privada, sin ánimo de lucro, que agrupa a 26 empresas promotoras de la Responsabilidad Social Corporativa, entre las que destacan: Mutualista Pichincha, Adelca, Diners, Microsoft, Pronaca, Telefónica Movistar y Petroamazonas.

Las tres últimas empresas mencionadas son ganadoras del premio "General Rumiñahui", otorgado por el Consejo Provincial de Pichincha en el año 2007, el cual consiste en un reconocimiento a las buenas prácticas de Responsabilidad Social Corporativa. Según Orquera et al (2013) "para estas empresas la RSC es un tema que va más allá de sus obligaciones legales, y en algunos casos la han adoptado como una política para poder operar"

En el estudio realizado por Orquera y otros (2013), destacan las prácticas de Responsabilidad Social Corporativa más importantes de estas empresas citadas; entre 
ellas menciona a Pronaca, empresa procesadora de alimentos y que se caracteriza por ser la principal de capital ecuatoriano con una clara y madura estrategia de RSC. Telefónica del Ecuador, desde su implantación en el mercado ecuatoriano, con su programa "PRONIÑO" se ha convertido en un referente en temas de RSC a nivel nacional, y se ha posicionado a nivel internacional con diferentes programas de éxito. Petroamazonas es la empresa de capital público que explota petróleo en suelo ecuatoriano, y que dada su delicada actividad se ha destacado por su buena gestión con sus grupos de interés.

Por otro lado, Torresano (2012), en su estudio sobre Responsabilidad Social de Empresas del Ecuador, efectuado en 2012 y aplicado a 743 empresas a nivel nacional, determinó que las empresas con prácticas de RSC, perciben como principales beneficios la existencia de mayor compromiso de colaboradores, gozar de una mejor imagen y reputación, un mayor fortalecimiento en la fidelidad de los clientes y consumidores y mejor relación con la comunidad. En virtud de lo cual, cerca del $42 \%$ de las empresas del país tienen una estrategia explícita de RSC.

Entre las empresas que consideran políticas y prácticas de RSC, el tema de mayor interés y sobre el cual se desarrollan acciones, es aquel relacionado con los derechos humanos; en este sentido, el $54 \%$ de los empresarios dispone de políticas dirigidas a sensibilizar sobre este tema; el $67 \%$ tiene políticas respecto a estos derechos y el $53 \%$ de las empresas posee políticas explícitas para evitar el relacionamiento con actores que violen la normativa legal (Torresano, 2012).

En cuanto al relacionamiento de las empresas con sus grupos de interés, el mismo estudio de Torresano (2012) identificó que, en referencia a los trabajadores; el 94\% de las empresas cuenta con políticas específicas de estabilidad laboral, el $70 \%$ muestra prácticas en seguridad y salud ocupacional y el 36\% de las empresas tiene más del $50 \%$ de mujeres como parte de su fuerza laboral.

Respecto al ambiente y desarrollo de la comunidad se determinó que el $37 \%$ de empresas desarrolla políticas de gestión ambiental, el 32\% tienen políticas de mitigación 
de impactos y $21 \%$ políticas de adaptación al cambio climático; sobre la relación con la comunidad, el 55\% de las empresas realizan aportes a la comunidad de diferentes formas: de modo directo (41\%), a través de la iglesia (10\%), a través de ONGs (7\%), y través de fundaciones de la empresa (4\%).

Como se puede observar, la RSC desde el año 2007 empieza a implementarse en Ecuador, sobre todo en las grandes empresas, las cuales enfocan sus esfuerzos principalmente en el ámbito de los derechos humanos, la generación de políticas de gestión ambiental y mitigación de impactos, y en menor medida al relacionamiento con las comunidades.

En definitiva, en relación a la Responsabilidad Social Corporativa en el Ecuador se ha logrado evidenciar la existencia de análisis de casos específicos, en sectores como el de hidrocarburos, turismo y educación universitaria.

\subsection{Caracterización del cantón Loja.}

La provincia de Loja, se encuentra ubicada en la Región Sur del Ecuador, junto a las provincias de El Oro y Zamora Chinchipe, esta provincia está conformada por 16 cantones y 92 parroquias, con una superficie de $10.994 .9 \mathrm{~km}^{2}$, de forma que constituye la provincia más extensa de la sierra ecuatoriana a excepción de Pichincha.

El cantón Loja, capital de la provincia del mismo nombre, se encuentra conformado por trece parroquias rurales y cuatro urbanas; y posee una superficie de $1882.83 \mathrm{~km}^{2}$. Ubicada en el noveno puesto de ciudades más pobladas del Ecuador, con un total de 214.855 habitantes, asentada el $67.70 \%$ en el área urbana y el $32.3 \%$ en el área rural y periferia.

El cantón posee una Población Económicamente Activa (PEA) de 91.978 personas, correspondiente al $43 \%$ de la población total. El $12.60 \%$ de dicha PEA se dedica principalmente a la agricultura y ganadería (sector primario); el 7.70\% trabaja en el sector 
secundario y el $71 \%$ en el sector terciario, el cual en los últimos años ha experimentado mayor crecimiento. (Plan de Desarrollo y Ordenamiento Territorial-PDyOT, 2010).

De acuerdo al Directorio de Empresas elaborado por el INEC (2013), en el cantón Loja existen 18.025 empresas, los cuales se distribuyen en los tres sectores económicos. En la figura 1 se aprecia que el sector con mayor representatividad es el terciario, conformado por 15.604 empresas; en segundo lugar se encuentra el sector secundario con 1.500 y el sector primario con 921 .

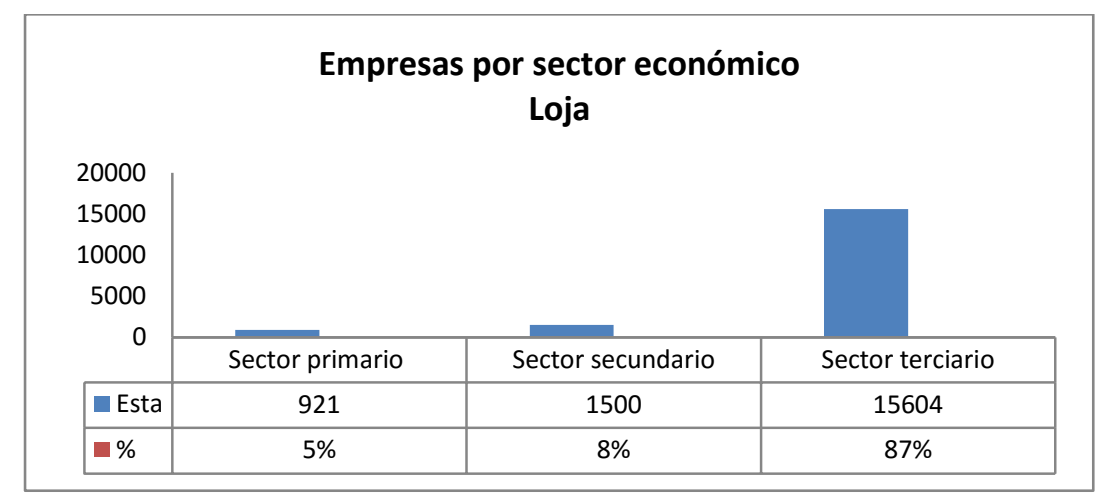

Figura 1. Empresas por sectores económicos en el cantón Loja, año 2013

Fuente: INEC (2013)

Como se observa en la figura 2 , el sector primario está divido en actividades de agricultura, ganaderia, silvicultura y pesca, y extracción de minas y canteras; el sector secundario, conformado por las industrias manufactureras; $y$, el sector terciario por servicios, comercio y construcción. 


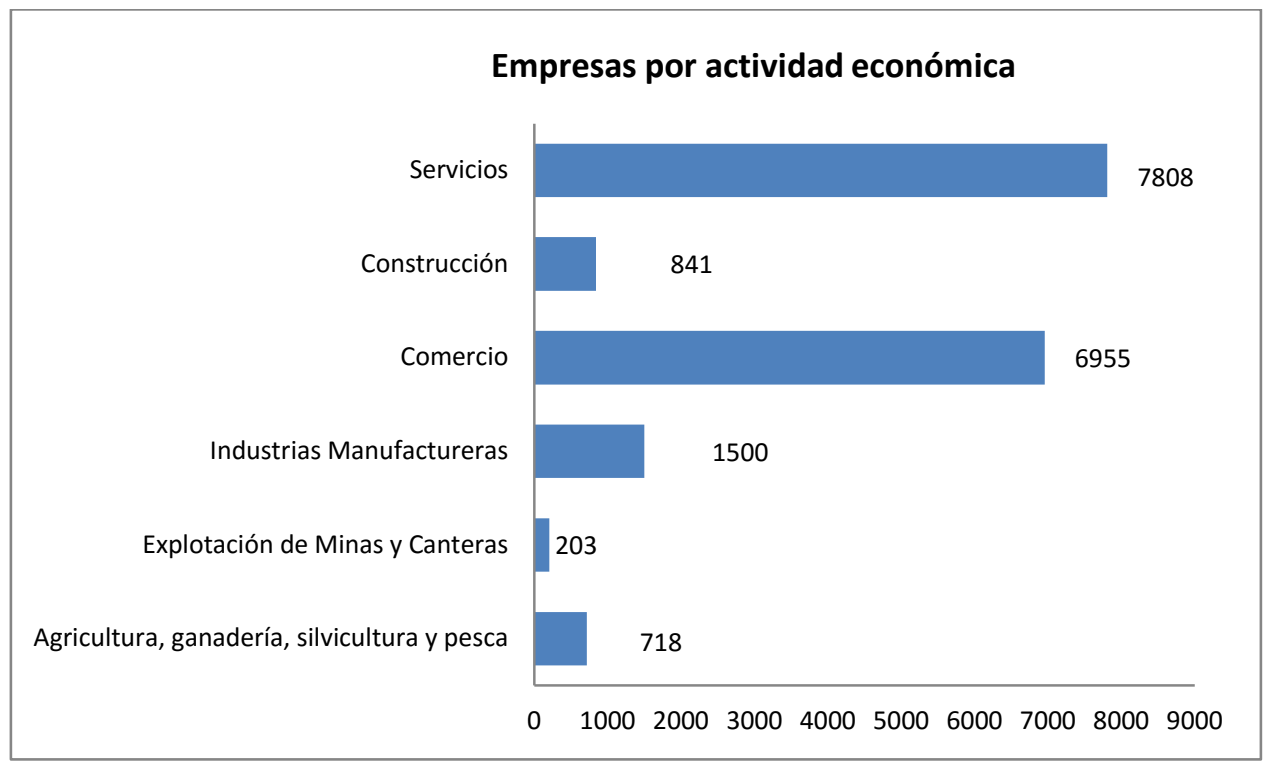

Figura 2. Empresas por actividad económica

Fuente: INEC (2013)

En su conjunto los tres sectores alcanzaron un nivel aproximado de ventas de 1.034 (millones de dólares), contribuyendo el sector terciario con el 92\% del total de ventas; el secundario con el $6 \%$ y el primario con el $2 \%$. De igual manera en su conjunto los tres sectores emplearon a 48.061 trabajadores de los cuales el $54 \%$ fueron hombres y el $46 \%$ mujeres.

En conclusión, el sector terciario es el que genera mayor crecimiento económica en el cantón Loja a través de la generación de empleo y recursos económicos; ahora bien, este escenario revela que la economía cantonal se sustenta en el sector servicios, de manera que el consumo se basa en la adquisición de productos provenientes de localidades externas al cantón y no de la producción local, principalmente de aquellos propios de la industria manufacturera y que el sector primario pierde cada vez más representatividad dentro de la actividad económica.

\section{OBJETIVOS}


Como consecuencia de la revisión de la literatura y el pertinente establecimiento del estado del arte sobre la cuestión abordada, se dotó a la investigación de los siguientes objetivos:

OBJ1. Definir el estado de la responsabilidad social corporativa en Ecuador.

OBJ2. Diagnosticar la situación actual de las empresas en cada uno de los sectores económicos en relación al conocimiento y aplicación de prácticas de responsabilidad social corporativa en el cantón Loja.

OBJ3. Determinar la relación existente entre la actividad de la empresa, el tamaño y la edad de la misma, con la aplicación de prácticas de responsabilidad social corporativa.

OBJ4. Clasificar las empresas según se aplica la responsabilidad social, tamaño y sector.

\section{HIPÓTESIS}

Una vez establecidos los Objetivos se delimitaron las hipótesis susceptibles de ser sometidas a contrastación y que son:

H1. La responsabilidad social en Ecuador es un concepto emergente con una importante impronta de desarrollo.

H2. La aplicación de prácticas de responsabilidad social no está condicionada por el tamaño de la empresa

H3. La edad de la empresa no influye decisivamente en la aplicación de prácticas de RSC.

H4. La actividad económica de la empresa incide en la relevancia que ésta otorga a sus diferentes grupos de interés.

\section{METODOLOGÍA}

La caracterización de los sectores económicos en el cantón Loja se realizó a través de la recopilación de información secundaría, con los últimos datos consolidados por el 
Instituto Nacional de Estadísticas y Censos de Ecuador (INEC), con información consolidada al 2013.

Se efectuó un estudio de caso, con carácter experimental y a modo de laboratorio, en el propio cantón Loja, con el objetivo de analizar la situación actual en torno a la aplicación de las prácticas de Responsabilidad Social Corporativa en los tres sectores de la economía.

Para la determinación de la RSC por sectores, fue aplicado un modelo de encuesta a una muestra compuesta por un total de 222 empresas, y calculada a través del método de muestreo aleatorio simple.

El instrumento empleado fue un cuestionario diseñado por el instituto ETHOS de Brasil y aplicado y validado por la Asociación de Empresas Cristianas (ADEC) de Paraguay, y el mismo fue ajustado a las características del campo de investigación. Dicho cuestionario se sometió a un proceso de depuración mediante la aplicación de test previos; finalmente, una vez cumplimentados los cuestionarios, se procedió a la validación de los mismos.

Con el citado instrumento se recopiló información de 23 indicadores por cada grupo de interés y respectivamente fueron evaluados de acuerdo al cumplimiento de las diferentes prácticas integradas en aquellos.

La comprobación de la hipótesis $\mathrm{H} 1$ se hizo a través de la revisión del estado del arte de la responsabilidad social en el Ecuador; las hipótesis $\mathrm{H} 2, \mathrm{H} 3$ y H4, se contrastaron por medio de un análisis de regresión lineal entre las variables objeto de estudio.

Para alcanzar los objetivos OBJ1 y OBJ4, se utilizó el método descriptivo a través del análisis de frecuencias y análisis clúster respectivamente.

\section{ANÁLISIS DE RESULTADOS}


Al aplicar el cuestionario de autodiagnóstico a las empresas de los tres sectores económicos, el $100 \%$ del sector primario conoce qué es la RSC y de los sectores secundario y terciario más del $80 \%$ de las empresas reconocen la RSC.

En relación a la aplicación de la RSC, todas las empresas de los sectores primario y secundario que si conocen qué es la RSC consideran que sí la aplican; y, en el sector terciario solo el $69 \%$ admite la aplicación de dichas prácticas.

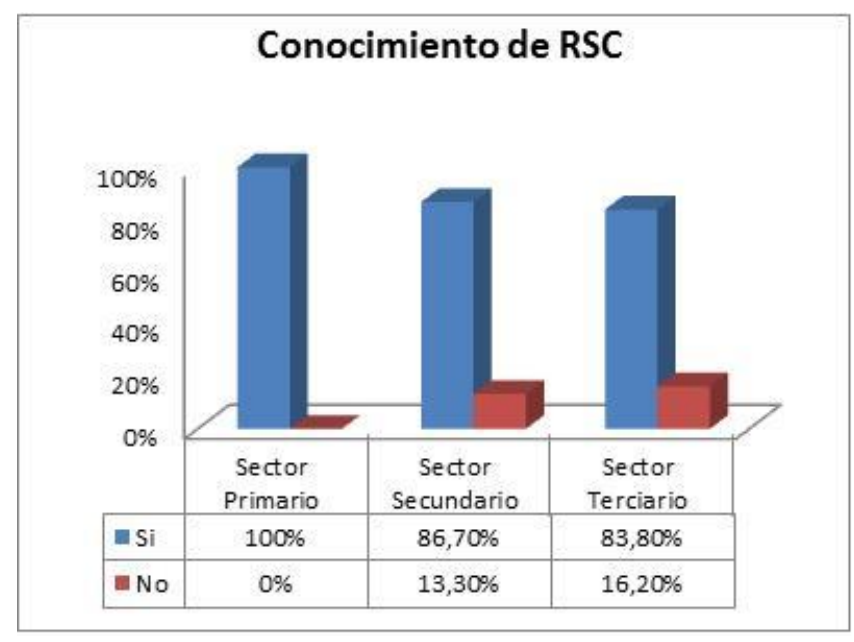

Figura 3. Conocimiento de la RSC

Fuente: Autores

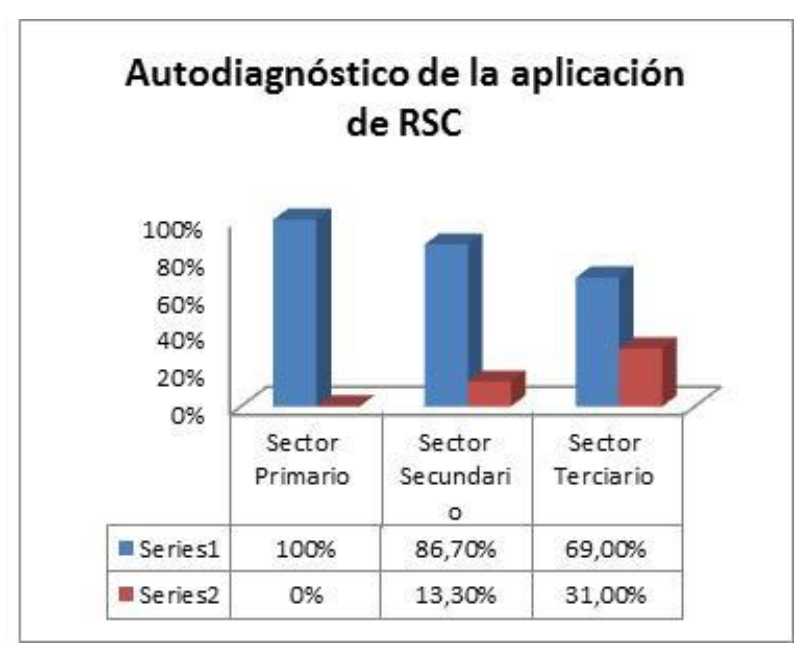

Figura 4. Autodiagnóstico de la aplicación de RSC Fuente: Autores

Para constatar la aplicación de la RSC en las empresas de los tres sectores se construyó un indicador general de acuerdo al cumplimiento de las prácticas que conforman cada uno de los 23 indicadores relacionados con gobierno corporativo y los diferentes grupos de interés. En el cuadro 1 se observa que el sector primario alcanza un $61.6 \%$ de cumplimiento de prácticas de RSC; el sector secundario el $53.1 \%$; y el terciario el $45.8 \%$. 
ESTUDIO EMPÍRICO SOBRE EL ESTADO Y TENDENCIAS DE LA RSC EN ECUADOR

Costa-Ruiz, Mónica

\begin{tabular}{|c|c|c|c|c|}
\hline $\begin{array}{l}\text { Gobierno Corporativo y } \\
\text { Grupos de interés }\end{array}$ & Indicadores & Primario & Secundario & Terciario \\
\hline \multirow{4}{*}{$\begin{array}{l}\text { Valores, transparencia y } \\
\text { Gobierno Corporativo }\end{array}$} & Indicador 1. Compromisos éticos & $77,1 \%$ & $76,2 \%$ & $69,2 \%$ \\
\hline & Indicador 2. Relaciones con la competencia & $33,3 \%$ & $31,1 \%$ & $29,0 \%$ \\
\hline & $\begin{array}{l}\text { Indicador 3. Diálogo e involucramiento de los grupos de } \\
\text { interés }\end{array}$ & $90,0 \%$ & $63,3 \%$ & $53,3 \%$ \\
\hline & $\begin{array}{l}\text { Indicador 4. Balance Social/ Memorias de RSE/ Reporte } \\
\text { de Sostenibilidad }\end{array}$ & $65,0 \%$ & $65,0 \%$ & $44,2 \%$ \\
\hline \multirow{4}{*}{ Público interno } & Indicador 5. Gestión participativa & $80,0 \%$ & $60,0 \%$ & $61,1 \%$ \\
\hline & Indicador 6. Compromiso con el desarrollo infantil & $36,7 \%$ & $40,0 \%$ & $33,8 \%$ \\
\hline & $\begin{array}{l}\text { Indicador 7. Compromiso con la no discriminación y } \\
\text { promoción de la equidad racial }\end{array}$ & $52,5 \%$ & $46,7 \%$ & $44,2 \%$ \\
\hline & $\begin{array}{l}\text { Indicador } 8 \text {. Compromiso con la promoción de la equidad } \\
\text { de género }\end{array}$ & $56,7 \%$ & $43,3 \%$ & $41,2 \%$ \\
\hline \multirow{4}{*}{ Medio ambiente } & $\begin{array}{l}\text { Indicador 9. Compromiso con el mejoramiento de la } \\
\text { calidad ambiental }\end{array}$ & $66,7 \%$ & $43,3 \%$ & $41,3 \%$ \\
\hline & Indicador 10. Educación y concienciación ambiental & $35,0 \%$ & $46,7 \%$ & $47,0 \%$ \\
\hline & $\begin{array}{l}\text { Indicador } 11 . \text { Gerenciamiento de los Impactos sobre el } \\
\text { medio ambiente y del ciclo de vida de productos y } \\
\text { servicios }\end{array}$ & $75,0 \%$ & $56,7 \%$ & $29,4 \%$ \\
\hline & $\begin{array}{l}\text { Indicador 12. Minimización de entradas y salidas de } \\
\text { insumos }\end{array}$ & $53,3 \%$ & $42,2 \%$ & $23,9 \%$ \\
\hline \multirow{2}{*}{ Proveedores } & $\begin{array}{l}\text { Indicador 13. Criterios de selección y evaluación de } \\
\text { proveedores }\end{array}$ & $68,0 \%$ & $52,0 \%$ & $45,0 \%$ \\
\hline & Indicador 14. Apoyo al desarrollo de proveedores & $67,5 \%$ & $63,3 \%$ & $50,0 \%$ \\
\hline \multirow{3}{*}{ Consumidores y clientes } & Indicador 15. Política de comunicación comercial & $62,5 \%$ & $53,3 \%$ & $55,3 \%$ \\
\hline & Indicador 16. Excelencia de la atención & $81,8 \%$ & $63,0 \%$ & $69,5 \%$ \\
\hline & $\begin{array}{l}\text { Indicador } 17 . \text { Conocimiento y gerenciamiento de los } \\
\text { daños potenciales de los productos y servicios }\end{array}$ & $96,0 \%$ & $77,3 \%$ & $69,1 \%$ \\
\hline \multirow{2}{*}{ Comunidad } & $\begin{array}{l}\text { Indicador 18. Gerenciamiento del impacto de la empresa } \\
\text { en la comunidad de entorno }\end{array}$ & $74,7 \%$ & $60,0 \%$ & $57,5 \%$ \\
\hline & Indicador 19. Financiamiento de la acción social & $56,0 \%$ & $40,0 \%$ & $34,1 \%$ \\
\hline \multirow{4}{*}{ Gobierno y sociedad } & $\begin{array}{l}\text { Indicador 20. Construcción de la ciudadanía por las } \\
\text { empresas }\end{array}$ & $26,7 \%$ & $46,7 \%$ & $28,3 \%$ \\
\hline & Indicador 21. Prácticas anticorrupción y anticoima & $90,0 \%$ & $68,9 \%$ & $70,2 \%$ \\
\hline & Indicador 22. Liderazgo e influencia social & $35,0 \%$ & $43,3 \%$ & $27,0 \%$ \\
\hline & $\begin{array}{l}\text { Indicador 23. Participación en proyectos sociales } \\
\text { gubernamentales }\end{array}$ & $36,7 \%$ & $37,8 \%$ & $29,6 \%$ \\
\hline & INDICADOR GENERAL & $61,6 \%$ & $53,1 \%$ & $45,8 \%$ \\
\hline
\end{tabular}

Cuadro 1. Índice general de RSC

Fuente: Autores

En el análisis de regresión, las variables tamaño, edad y sector al que pertenece la empresa, no son significativas desde un punto de vista estadístico, ya que no rechazamos el contraste t de Student, de que el coeficiente de la variable es igual a 0, con una probabilidad del 99\%. Los resultados se generaron en el programa SPSS, mediante una regresión logit donde la variable endógena binaria es:

1 (Si aplica RSC)

0 (No aplica RSC) 
ESTUDIO EMPÍRICO SOBRE EL ESTADO Y TENDENCIAS DE LA RSC EN ECUADOR

Costa-Ruiz, Mónica

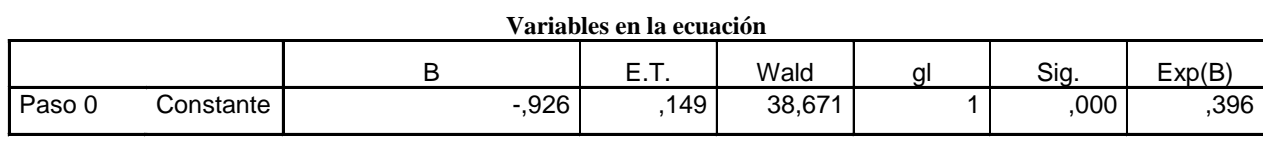

\begin{tabular}{|c|c|c|c|c|c|}
\hline & & & & \\
\hline \multirow{14}{*}{ Paso 0} & \multirow{13}{*}{ Variables } & Tamaño Empresa & 5,047 & 3 & 168 \\
\hline & & Tamaño_Empresa(1) & ,950 & 1 & ,330 \\
\hline & & Tamaño_Empresa(2) & 4,061 & 1 & 044 \\
\hline & & Tamaño_Empresa(3) & 997 & 1 & 318 \\
\hline & & Edad_empresa & 2,795 & 5 & ,732 \\
\hline & & Edad_empresa(1) & 195 & 1 & ,659 \\
\hline & & Edad_empresa(2) & 144 & 1 & ,704 \\
\hline & & Edad_empresa(3) & ,071 & 1 & ,790 \\
\hline & & Edad_empresa(4) & 177 & 1 & 674 \\
\hline & & Edad_empresa(5) & 1,614 & 1 & 204 \\
\hline & & Sector & 6,281 & 2 & ,043 \\
\hline & & Sector(1) & 4,149 & 1 & ,042 \\
\hline & & Sector(2) & 1,792 & 1 & 181 \\
\hline & \multicolumn{2}{|c|}{ Estadísticos globales } & 11,864 & 10 & 294 \\
\hline
\end{tabular}

\section{Cuadro 2. Regresión lineal}

Fuente: Autores

En teoría, al revisar que los factores empresariales no explican la actuación de las empresas en torno a la RSC, se realizó una clasificación de las empresas mediante un análisis cluster bietápico, donde se tuvo en cuenta que las variables a utilizar son discretas. Las variables empleadas son: tamaño de la empresa, aplicación de prácticas de RSC y sector económico.

Se generaron 6 conglomerados a partir de la clasificación de las empresas, según las 3 variables citadas anteriormente. 


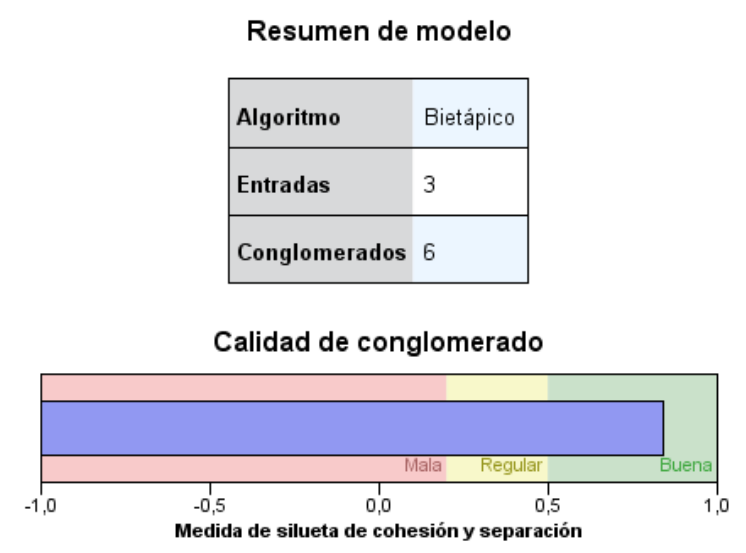

Cuadro 3. Resumen del modelo cluster

Fuente: Autores

Las empresas se clasificaron en: 1) empresas pequeñas de servicios sin sostenibilidad social; 2) microempresa de servicio sin conciencia social; 3) microempresas del sector industrial con prácticas sociales; 4) microempresas de servicios con prácticas sociales; 5) empresas medianas y grandes del sector servicios con conciencia social; 6) pequeña empresa del sector servicios con conciencia social.

\section{Conglomerados}

Importancia de entrada (predictor) $\square 1,0 \square 0,8 \square 0,6 \square 0,4 \square 0,2 \square 0,0$

\begin{tabular}{|c|c|c|c|c|c|c|}
\hline Conglomerado & 4 & 6 & \multirow[t]{2}{*}{2} & \multirow[t]{2}{*}{1} & \multirow[t]{2}{*}{3} & 5 \\
\hline \begin{tabular}{|l|l} 
Etiqueta \\
\end{tabular} & & & & & & \\
\hline \multicolumn{7}{|l|}{ Descripción } \\
\hline Tamaño & $\begin{array}{r}27.9 \% \\
(62)\end{array}$ & $\begin{array}{r}22.5 \% \\
(50)\end{array}$ & $\begin{array}{r}14.0 \% \\
(31)\end{array}$ & $\begin{array}{r}13.5 \% \\
(30)\end{array}$ & $\mid \begin{array}{r}11.3 \% \\
(25)\end{array}$ & $\begin{array}{r}10.8 \% \\
(24)\end{array}$ \\
\hline \multirow[t]{2}{*}{ Entradas } & $\begin{array}{c}\text { Tamaño de la } \\
\text { empresa } \\
\text { Microempresa } \\
(100.0 \%)\end{array}$ & $\begin{array}{c}\text { Tamaño de la } \\
\text { empresa } \\
\text { Pequeña (100.0\%) }\end{array}$ & $\begin{array}{c}\text { Tamaño de la } \\
\text { empresa } \\
\text { Microempresa } \\
(74.2 \%)\end{array}$ & $\begin{array}{c}\text { Tamaño de la } \\
\text { empresa } \\
\text { Pequeña }(100.0 \%)\end{array}$ & $\begin{array}{c}\text { Tamaño de la } \\
\text { empresa } \\
\text { Microempresa } \\
(44.0 \%)\end{array}$ & $\begin{array}{c}\text { Tamaño de la } \\
\text { empresa } \\
\text { Mediana ( } 87.5 \%)\end{array}$ \\
\hline & $\begin{array}{c}\text { Sector al que } \\
\text { pertenece la } \\
\text { empresa } \\
\text { Terciario }(100.0 \%)\end{array}$ & $\begin{array}{c}\text { Sector al que } \\
\text { pertenece la } \\
\text { empresa } \\
\text { Terciario }(100.0 \%)\end{array}$ & $\begin{array}{c}\text { Sector al que } \\
\text { pertenece la } \\
\text { empresa } \\
\text { Terciario }(100.0 \%)\end{array}$ & $\begin{array}{c}\text { Sector al que } \\
\text { pertenece la } \\
\text { empresa } \\
\text { Terciario }(100.0 \%)\end{array}$ & $\begin{array}{c}\text { Sector al que } \\
\text { pertenece la } \\
\text { empresa } \\
\text { Secundario }(60.0 \%)\end{array}$ & $\begin{array}{c}\text { Sector al que } \\
\text { pertenece la } \\
\text { empresa } \\
\text { Terciario }(100.0 \%)\end{array}$ \\
\hline
\end{tabular}

REDMARKA UIMA-Universidad de A Coruña - CIECID

Año VIII, Número 15, (2015), v I pp. 43-67

http://www.redmarka.net/ ISSN 1852-2300 
Cuadro 4. Conglomerados

Fuente: Autores

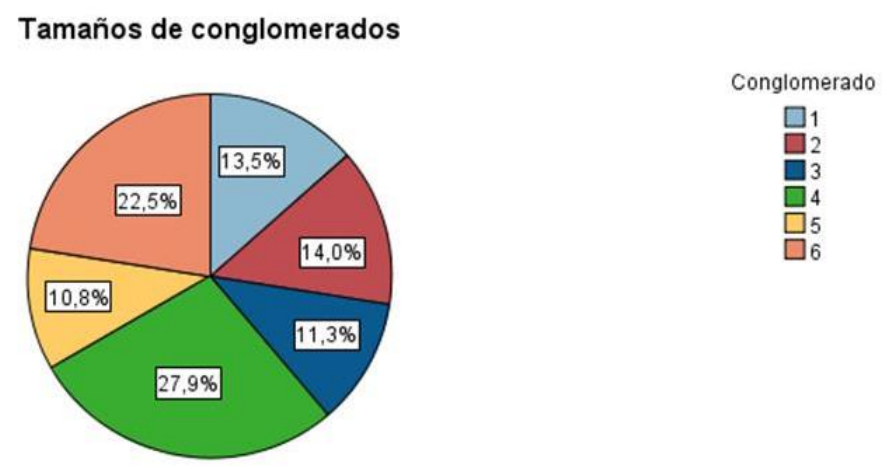

\begin{tabular}{|c|c|}
\hline $\begin{array}{l}\text { Tamaño de } \\
\text { conglomerado más } \\
\text { pequeño }\end{array}$ & $24(10.8 \%)$ \\
\hline $\begin{array}{l}\text { Tamaño de } \\
\text { conglomerado más } \\
\text { grande }\end{array}$ & $62(27.9 \%)$ \\
\hline $\begin{array}{l}\text { Cociente de tamaños: } \\
\text { Conglomerado más } \\
\text { grande a conglomerado } \\
\text { más pequeño }\end{array}$ & 2.58 \\
\hline
\end{tabular}

Cuadro 5. Conglomerado

Fuente: Autores

Se observa que el cluster microempresas de servicios con prácticas sociales, es el tipo de empresa más común en Loja; seguido del cluster pequeña empresa del sector servicios con conciencia social, con lo cual podemos afirmar que las empresas de servicios de menor tamaño son las más concienciadas respecto a la RSC; por su parte las microempresas del sector industrial con prácticas sociales son las menos frecuentes.

En función del análisis cluster, se puede proponer realizar campañas de concienciación con las empresas pequeñas de servicios sin sostenibilidad social y microempresa de servicio sin concienciación social, que no aplican de forma extensiva la RSC a fin de generar mejores prácticas de RSC en la zona de estudio. 


\section{CONCLUSIONES}

La Responsabilidad Social Corporativa en Ecuador es un concepto de reciente aplicación que toma importancia y genera conciencia en las empresas, respecto a su vinculación con los grupos de interés en miras de lograr un equilibro entre lo económico, social y ambiental, para procurar con ello el desarrollo económico sostenible.

Las empresas de los tres sectores económicos del cantón Loja tienen un alto nivel de conocimiento de lo qué es la RSC y consideran que aplican diferentes prácticas de la misma con sus grupos de interés.

Del análisis realizado se puede concluir que el sector donde en mayor medida se aplican prácticas de Responsabilidad Social Corporativa, según el indicador general, es el sector primario.

Las variables, edad, tamaño y sector económico no son factores determinantes en la aplicabilidad de la RSC. Por lo que luego de contrastar las hipótesis planteadas se evidencia que las hipótesis $\mathrm{H} 2$ y H3 se cumplen y la H4 se refuta.

De acuerdo con el análisis cluster, a pesar de ser el sector primario el que mayor cumplimiento del índice general de RSC tiene, el cluster de mayor frecuencia en el cantón Loja es el constituido por las microempresas del sector servicio con conciencia social y el de menor frecuencia son las microempresas del sector industrial con prácticas sociales.

\section{BIBLIOGRAFÍA}

- Argandoña, A. (2012). ¿Qué es y qué no es la responsabilidad social? IESE Business School-Universidad de Navarra: Madrid- España

- Bateman, T. y Snell, S. (2004). Administración Un nuevo panorama competitivo. México: Mc Graw Hill 
- Camejo, A. (2010). Ética y Responsabilidad Social en las Relaciones Laborales. Valencia, España: Fundación Universitaria Andaluza Inca Garcilaso

- Comisión de las comunidades europeas (2001). Libro verde, fomentar un marco europeo para la responsabilidad social de las empresas. [Recuperado 17-04-2015] de https://ec.europa.eu/research/era/pdf/era_gp_final_es.pdf

- Diez de Castro, E. y otros (2001). Administración y dirección. Madrid: Mc Graw Hill.

- García, J. (2012). La Responsabilidad de las organizaciones desde la perspectiva de la demanda. [Recuperado 17-04-2015] de http://buleria.unileon.es/bitstream/handle/10612/2685/tesis_d38e3ahelena.pdf?seq uence

- Lorenzo, J. (2007). Ética y gestión ética: nuevos retos gerenciales. Revista Gestión: Capital Humano, No. 213, Septiembre 2007

- Martínez, P. y Rodríguez, I. (2013). Revisión teórica del concepto y estrategias de medición de la responsabilidad social corporativa. Revista de ciencias social Prisma Social Nro. 11.

- Orquera, M. y otros (2013). La Responsabilidad Social Corporativa en economías en desarrollo: Una visión de la realidad ecuatoriana. [Recuperado 17-03-2015] de http://www.revistaespacios.com/a14v35n02/14350206.html

- Raufflet, E. (2012). Responsabilidad Social Empresarial. México: Pearson.

- Tejedor, J. (2015). La Responsabilidad social corporativa y su aporte a la economía. [Recuperado 15-04-2015] de http://www.researchgate.net/publication/273513892

- Torresano, M. (2012). Responsabilidad social de empresas del Ecuador 2012. [Recuperado 05-04-2015] de http://investiga.ide.edu.ec/images/pdfs/2012diciembre/analisis.pdf 